\title{
Is COVID-19 that different in hemodialysis patients?: A single-center experience
}

\author{
Marina Reis* iD, Catarina Almeida* iD, Ana Ventura (iD), Catarina Ribeiro iD, Ana Marta Gomes (iD), \\ Daniela Lopes iD, Clara Santos iD, Sónia Sousa iD, João Carlos Fernandes iD \\ ${ }^{1}$ Nephrology Department, Centro Hospitalar Vila Nova de Gaia/Espinho, Vila Nova de Gaia, Portugal \\ *Both authors equally contributed to this work
}

\section{ABSTRACT}

Coronavirus disease 2019 (COVID-19) has affected millions worldwide, and in particular the care of patients on maintenance hemodialysis. These patients are thought to be at high risk of severe SARS-CoV-2 infection due to their older age and multiple comorbidities. The aim of this study was to compare hemodialysis and non-dialysis COVID-19 patients and find possible risk factors for mortality in hemodialysis patients.

We developed a single-center retrospective cohort study, from March $1^{\text {st }}$ to December $31^{\text {st }}, 2020$, that included maintenance hemodialysis patients hospitalized with laboratory confirmed SARS-CoV-2 infection, and age and sex propensity matched non-dialysis patients also hospitalized with a laboratory confirmed SARS-CoV-2 infection (1:1).

A total of 34 hemodialysis patients were included, $70.6 \%$ male, mean age 76.5 years and on maintenance hemodialysis for 3.0 [0.5-23] years. At admission, 50.0\% needed oxygen supply. Median hospital stay duration was 11.0 [5.8-17.0] days, and 38.2\% developed bacterial superinfection. Maintenance hemodialysis patient mortality rate was $32.4 \%$. When matched to the non-dialysis group, the hemodialysis group developed more often respiratory insufficiency $(50.0 \%$ vs $8.8 \%, p<0.001)$ and had higher ferritin $(1658.0$ vs $623.5, p=0.004)$ and troponin $T$ (130.0 vs 31.0, $p<0.001$ ) levels, whereas the non-dialysis group had higher transaminases levels. There was no statistical difference regarding hospitalization time, bacterial superinfection, or mortality between groups. When the logistic regression was performed, only bacterial superinfection was a predictor for mortality in hemodialysis COVID-19 patients (0.01 [0.00-0.26]).

There was no difference in hospital stay nor in death rate between hemodialysis and non-dialysis COVID-19 patients. Despite these results, we must emphasize that mortality in the dialysis group was particularly high, with up to $32 \%$ of in-hospital mortality, and that bacterial superinfection has been shown to be an independent predictor of mortality. These results highlight the importance of interventions, such as full vaccination coverage, to mitigate the burden of COVID-19 in hemodialysis patients.

Keywords: chronic hemodialysis; COVID-19; mortality

(C) 2021 Portuguese Journal of Nephrology \& Hypertension. Published by Publicações Ciência \& Vida This is an open access article under the CC BY-NC-ND license (http://creativecommons.org/licenses/by-nc-nd/4.0/).

\section{INTRODUCTION}

Coronavirus disease 2019 (COVID-19) due to severe acute respiratory syndrome coronavirus 2 (SARS-CoV-2) infection has affected millions worldwide, and in particular the care of patients on maintenance hemodialysis (HD). In Portugal, from March $2^{\text {nd }}$ until the end of the year of 2020 , a total of 413,678 people were infected, with up to 6,906 deaths. ${ }^{1}$ Several clinical studies and meta-analysis have demonstrated that the elderly and patients with comorbidities, such as diabetes mellitus, hypertension, cardiovascular disease, pulmonary chronic disease, cancer and chronic kidney disease (CKD) are prone to develop severe disease and have poorer outcomes. ${ }^{2-6}$ The earlier published reports found that HD patients presented with less symptoms and a milder course, often asymptomatic, whereas more recent papers have shown that clinical presentation is similar to the general population. ${ }^{2-4,7-10}$ Patients on HD are at particular high risk for severe COVID-19 infection due to their old age, multiple comorbidities, and relative immunosuppression due to uremia. Another contributor for the greater risk is inherent to the dialysis treatment, since there is frequent contact with other patients, even though preventive and isolation measures were implemented at the dialysis facilities at the beginning of the COVID-19 outbreak. ${ }^{7}$ In fact, the mortality rates that have been reported are far higher than in the general population, with the European Renal Association and Transplant Association Registry reporting $20 \%$ mortality rate among patients with end-stage kidney disease. ${ }^{7}$ However, evidence on particular features of COVID-19 as well as on predictors for mortality in HD patients is still lacking.

The aim of our study was to compare the clinical presentation, laboratory and radiology data, and outcomes between HD and nondialysis COVID-19 hospitalized patients and find possible risk factors for mortality in HD patients. 


\section{MATERIAL AND METHODS}

\section{Population Selection and Study Design}

We conducted a single-center retrospective cohort study from $1^{\text {st }}$ March 2020 to $31^{\text {st }}$ December 2020 including all patients on maintenance HD admitted to Centro Hospitalar Vila Nova de Gaia/ Espinho with a confirmed SARS-CoV-2 infection. Asymptomatic patients and those admitted only for public health reasons were excluded. We used propensity score matching (without the use of a caliper width) to adjust for age and sex, to compare HD patients to a control group of non-dialysis patients hospitalized with a confirmed SARS-CoV-2 infection (1:1). All patients enrolled in this study had a confirmed SARS-CoV-2 infection diagnosed by real time PCR SARS-CoV-2 test.

\section{Data Collection}

Data regarding patients' baseline characteristics, symptoms, laboratory, and radiology findings at presentation, length of hospital stay, treatment regimens (antimalarial and antiviral agents, glucocorticoids and ventilatory support), clinical outcomes, comorbidities and use of angiotensin converting enzyme inhibitor/angiotensin receptor blockers (ACEi/ARB) were collected by consulting electronic medical records. Obesity was defined as a body mass index superior to $30 \mathrm{Kg} / \mathrm{m}^{2}$. The Katz index was used to determine the degree of autonomy in activities of daily living based on data from the clinical process: a score of above 4 was "independent", a score of 3 or 4 was "partially dependent" and below 3 "dependent". Bacterial superinfection was defined as a positive bacterial culture from a lower respiratory tract specimen (sputum, bronchial/tracheal aspirates or bronchoalveolar lavage).

\section{Clinical Management}

In respect to therapy, from March to August 2020, hydroxychloroquine and lopinavir/ritonavir was given to patients with $\mathrm{PaO} 2 / \mathrm{FiO} 2<300$ or with radiological evidence of pneumonia unless they had prolonged QT on electrocardiography.

From September 2020 until December 2020, patients were treated with intravenous dexamethasone if they needed supplementary oxygen or ventilation or if they had acute respiratory distress syndrome or septic shock. COVID-19 patients were treated with remdesivir if they were within the first six days of symptoms and if they needed supplementary oxygen and had no absolute contraindication (transaminases above 5 times the normal range and glomerular filtration rate inferior to $30 \mathrm{~mL} / \mathrm{min}$ ).

\section{Ethical concerns}

Our research was conducted in full accordance with the World Medical Association Declaration of Helsinki. Informed consent was waived as part of the public health outbreak investigation.

\section{Statistical analysis}

Categorical variables were presented as frequencies and percentages and were compared with the use of Fisher's exact test or the chi-square test, as appropriate. Continuous variables were presented as means and standard deviations, or medians and $25^{\text {th }}$ and $75^{\text {th }}$ percentile for variables with skewed distributions. Normal distribution was checked using Kolmogorov-Smirnov test or skewness and kurtosis. Continuous normally distributed variables were compared with the use of Student's t-test and continuous variables with non-normal distribution were compared with the Mann-Whitney test. Kaplan-Meier estimates were used to construct the survival curves. Bivariate logistic regression was used to find mortality predictors of HD patients. All reported $p$ values are two-tailed, with a $p$ value of 0.05 indicating statistical significance. Statistical analyses were performed by SPSS for Windows software version 23.0 (SPSS Inc., Chicago, IL).

\section{RESULTS}

In total, 34 maintenance HD patients were admitted at our center with COVID-19 and were age and gender matched to 34 non-dialysis patients. Median time on maintenance HD was 3.0 [0.5-23] years. Table I illustrates the comparison between maintenance HD patients and the control group. Baseline characteristics and comorbidities were similar between groups, except for peripheral arterial disease that was more frequent in HD patients ( $20.6 \%$ vs $2.9 \%, p=0.024)$ and cerebrovascular disease that was more common in the control group (17.6\% vs 52.9\%, p=0.002). There was no difference in the use of ACEi/ ARB. COVID-19 symptoms were similar between groups, with fever the most frequent symptom, present in $67.6 \%$ of patients. However, more patients in the HD group presented with respiratory insufficiency and needed supplementary oxygen $(50.0 \%$ vs $8.8 \%, p<0.001)$.

Patients in the HD group had higher procalcitonin (1.3 vs 0.3 $p=0.007$ ), ferritin ( 1658.0 vs $623.5, p=0.004)$ and troponin $T$ ( 130.0 vs $31.0, p<0.001$ ) levels versus the control group. Transaminases were higher in the control group ( 32.0 vs $27.5, p=0.039 ; 20.0$ vs 24.5 , $p=0.046)$.

Unilateral pneumonia in the chest $X$-ray was more frequent in the control group ( $11.8 \%$ vs $32.4 \%, p=0.041)$.

Therapeutic options and clinical outcomes (hospital stay, bacterial superinfection, admission to intensive care unit (ICU) and mortality) were similar in both groups.

Maintenance HD and control group presented similar survival curves (log rank = 0.448. $p=0.503$ ) (Figure 1 ).

Table II compares maintenance HD patients who died during the hospitalization and discharged patients. The large majority $(91.7 \%$, $\mathrm{n}=11$ ) of HD patients with an indication to ICU admission were refused. Leucocyte (5.2 vs $7.7, p=0.004$ ) and neutrophil (4.0 vs $6.5, p=0.008$ ) count were higher in the mortality group. Bacterial superinfection was also more frequent in the in-hospital death group. After adjusting for covariables, only bacterial superinfection was a predictor of inhospital mortality for HD patients (OR 0.01 [0.00-0.26]). 


\section{Table I}

Comparison between baseline characteristics, comorbidities, laboratory data and radiology findings and clinical outcomes between chronic hemodialysis and control group.

\begin{tabular}{|c|c|c|c|c|}
\hline & All patients $(\mathrm{N}=68)$ & HD patients $(\mathrm{N}=34)$ & Control group ( $\mathrm{N}=34$ ) & p-value \\
\hline Male sex, n (\%) & $45(66.2)$ & $24(70.6)$ & $21(61.8)$ & 0.442 \\
\hline Age, years, mean $\pm S D$ & $76.3 \pm 11.4$ & $76.5 \pm 12.3$ & $76.0 \pm 10.7$ & 0.859 \\
\hline \multicolumn{5}{|l|}{ Katz } \\
\hline Independent & $35(51.5)$ & $17(50.0)$ & $18(52.9)$ & 0.808 \\
\hline Partially dependent & $21(30.9)$ & $14(41.2)$ & $7(20.6)$ & 0.066 \\
\hline Dependent & $12(17.6)$ & $3(8.8)$ & $9(26.5)$ & 0.056 \\
\hline Diabetes Mellitus, n (\%) & $35(51.4)$ & $16(47.1)$ & 19(55.9) & 0.134 \\
\hline Hypertension, n (\%) & $54(79.4)$ & $29(85.3)$ & $25(73.5)$ & 0.230 \\
\hline Heart Failure, n (\%) & $30(44.1)$ & $16(47.1)$ & $14(41.2)$ & 0.625 \\
\hline Peripheral arterial disease, $\mathrm{n}(\%)$ & $8(11.8)$ & $7(20.6)$ & $1(2.9)$ & 0.024 \\
\hline Cerebrovascular disease, n (\%) & $24(35.3)$ & $6(17.6)$ & $18(52.9)$ & 0.002 \\
\hline Pulmonary chronic disease, $n$ (\%) & $11(16.2)$ & $7(20.6)$ & $4(11.8)$ & 0.323 \\
\hline Cancer, n (\%) & $12(17.6)$ & $8(23.5)$ & $4(11.8)$ & 0.203 \\
\hline RAAS inhibitor, n (\%) & $22(32.4)$ & $9(26.5)$ & $13(38.2)$ & 0.300 \\
\hline \multicolumn{5}{|l|}{ Symptoms } \\
\hline Fever, $\mathrm{n}(\%)$ & $46(67.6)$ & $23(67.6)$ & $23(67.6)$ & 1.000 \\
\hline Cough, n (\%) & $36(52.9)$ & $18(52.9)$ & $18(52.9)$ & 1.000 \\
\hline Shortness of breath, $n$ (\%) & $43(63.2)$ & $21(61.8)$ & $22(64.7)$ & 0.801 \\
\hline Myalgias, $n$ (\%) & $8(11.8)$ & $4(11.8)$ & $4(11.8)$ & 1.000 \\
\hline Gastrointestinal, n (\%) & $10(14.7)$ & $4(11.8)$ & $6(17.6)$ & 0.493 \\
\hline Days of symptoms up to hospitalization, median (IQR) & $3.0(1.0-5.0)$ & $3.0(1.0-4.0)$ & $3.0(1.0-6.0)$ & 0.622 \\
\hline \multicolumn{5}{|l|}{ Ventilatory support at admission } \\
\hline Not needed, $n(\%)$ & $38(55.9)$ & $15(44.1)$ & $23(67.6)$ & 0.051 \\
\hline $\mathrm{FiO} 2<36 \%, \mathrm{n}(\%)$ & $20(29.4)$ & $17(50.0)$ & $3(8.8)$ & $<0.001$ \\
\hline $\mathrm{FiO} 2>36 \%, \mathrm{n}(\%)$ & $2(2.9)$ & $0(0)$ & $2(5.9)$ & 0.493 \\
\hline Non-invasive ventilation, $n(\%)$ & $2(2.9)$ & $1(2.9)$ & $1(2.9)$ & 1.000 \\
\hline Mechanic ventilation, $n$ (\%) & $6 / 8.8)$ & $1(2.9)$ & $5(14.7)$ & 0.197 \\
\hline \multicolumn{5}{|l|}{ Admission laboratory data } \\
\hline Leucocyte count, x10E3/uL, median (IQR) & $6.1(4.7-8.2)$ & $6.0(4.3-7.8)$ & $6.15(4.9-10.9)$ & 0.317 \\
\hline Lymphocyte count, /uL, median (IQR) & $985.0(622.5-1365.0)$ & $795(460-1317.5)$ & 1050 (717.5-1387.5) & 0.084 \\
\hline Neutrophil count, x10E3/uL, median (IQR) & $4.4(3.0-6.5)$ & $4.4(2.9-6.4)$ & $4.3(3.1-8.5)$ & 0.778 \\
\hline Platelet count, $x 10 E 3 / \mathrm{uL}$, mean $\pm \mathrm{SD}$ & $171.5 \pm 76.0$ & $157.2 \pm 76.6$ & $186.1 \pm 73.7)$ & 0.121 \\
\hline Aspartate transaminase, $\mathrm{U} / \mathrm{L}$ - median (IQR) & $32(23-48)$ & $27.5(21.8-38.5)$ & $41(27.5-48.5)$ & 0.039 \\
\hline Alanine transaminase, $\mathrm{U} / \mathrm{L}$ - median (IQR) & $22(16-31)$ & $20.0(13-28)$ & $24.5(17.8-38.3)$ & 0.046 \\
\hline $\mathrm{LDH}, \mathrm{U} / \mathrm{L}$, median (IQR) & $270(224-346)$ & $255(225-315)$ & $318(222.8-386.3)$ & 0.147 \\
\hline CRP, mg/dL, median (IQR) & $7.6(3.2-15.4)$ & $6.3(3.1-13.6)$ & $9.7(2.9-17.3)$ & 0.506 \\
\hline Procalcitonin, $\mathrm{ng} / \mathrm{mL}$, median (IQR) & $0.5(0.2-1.6)$ & $1.3(0.4-2.3)$ & $0.290 .6-9.1)$ & 0.007 \\
\hline Ferritin, ng/mL, median (IQR) & $1251(623.5-1972.0)$ & $1658(1249.5-2533.0)$ & $623.5(266.8-1398.5)$ & 0.004 \\
\hline D-dimer, ug/mL median (IQR) & $1.7(1.2-3.5)$ & $1.7(1.5-3.8)$ & $1.7(0.8-2.8)$ & 0.337 \\
\hline PT, seconds, median (IQR) & $15.3(14.0-16.9)$ & $15.1(13.7-16.6)$ & $15.9(14.1-17.1)$ & 0.304 \\
\hline Troponin T, ng/mL, median (IQR) & $88.5(35.0-149.3)$ & $130.0(86.5-199.5)$ & $31(12-72.8)$ & $<0.001$ \\
\hline CK. U/L, median (IQR) & $103.5(47.5-182.0)$ & $88.5(39.8-197.8)$ & $110(70.8-175.3)$ & 0.351 \\
\hline \multicolumn{5}{|l|}{ Radiology findings } \\
\hline Unilateral pneumonia, $n(\%)$ & $15(22.1)$ & $4(11.8)$ & $11(32.4)$ & 0.041 \\
\hline Bilateral pneumonia, $n$ (\%) & $10(14.7)$ & $3(8.8)$ & $7(20.6)$ & 0.171 \\
\hline \multicolumn{5}{|l|}{ Treatment } \\
\hline Hydroxychloroquine, $n(\%)$ & $32(47.1)$ & $17(50)$ & 15(44.1) & 0.627 \\
\hline Antiviral (Lopinavir/ritonavir or Remdesivir), $\mathrm{n}(\%)$ & $14(20.6)$ & $5(14.7)$ & $9(26.5)$ & 0.230 \\
\hline Glucocorticoid, n (\%) & $8(11.8)$ & $4(11.8)$ & $4(11.8)$ & 1.000 \\
\hline \multicolumn{5}{|l|}{ Clinical outcomes } \\
\hline Bacterial superinfection, $n(\%)$ & $20(29.4)$ & $13(38.2)$ & $7(20.6)$ & 0.110 \\
\hline Hospitalization time (days), median (IQR) & $9(5.3-16.8)$ & $11.0(5.8-17.0)$ & $7.0(5.0-16.3)$ & 0.222 \\
\hline Admission to ICU $-n(\%)$ & $6(8.8)$ & $1(2.9)$ & $5(14.7)$ & 0.197 \\
\hline Death $-\mathrm{n}(\%)$ & $23(33.8)$ & $11(32.4)$ & $12(35.3)$ & 0.798 \\
\hline
\end{tabular}

CK - creatinine kinase; CRP - C reactive protein; HD - hemodialysis; ICU - intensive care unit; IQR - interquartile range; LDH - lactate dehydrogenase; PT - prothrombin time; RAAS - renin angiotensin aldosterone; SD - standard deviation. 


\section{Table II}

Predictors of mortality on chronic hemodialysis patients infected with SARS-CoV-2.

\begin{tabular}{|c|c|c|c|c|}
\hline & Discharged patients $(\mathrm{N}=23)$ & In-hospital death $(\mathrm{N}=11)$ & $\mathrm{p}$-value & OR [IC 95\%] \\
\hline Male sex. n (\%) & $17(73.9)$ & $7(63.6)$ & 0.692 & \\
\hline Age, years, mean $\pm S D$ & $75.8 \pm 13.2$ & $77.9 \pm 10.6$ & 0.651 & \\
\hline \multicolumn{5}{|l|}{ Katz } \\
\hline Independent, $\mathrm{n}(\%)$ & $13(56.5)$ & $4(36.4)$ & 0.271 & \\
\hline Partially dependent, $n$ (\%) & $7(30.4)$ & $7(63.6)$ & 0.135 & \\
\hline Dependent, $n$ (\%) & $3(13.0)$ & $0(0.0)$ & 0.535 & \\
\hline Diabetes Mellitus, n (\%) & $10(43.5)$ & $6(54.5)$ & 0.545 & \\
\hline Hypertension, $n$ (\%) & $18(78.3)$ & $11(100.0)$ & 0.150 & \\
\hline Heart Failure, $n(\%)$ & $11(47.8)$ & $5(45.5)$ & 0.897 & \\
\hline Peripheral arterial disease, $n(\%)$ & $5(21.7)$ & $2(18.2)$ & 1.000 & \\
\hline Cerebrovascular disease, $n(\%)$ & $4(17.4)$ & $2(18.2)$ & 1.000 & \\
\hline Pulmonary chronic disease, $n(\%)$ & $5(21.7)$ & $2(18.2)$ & 1.000 & \\
\hline Malignancy, n (\%) & $4(17.4)$ & $4(36.4)$ & 0.388 & \\
\hline RAAS inhibitor, $\mathrm{n}(\%)$ & $7(30.4)$ & $2(18.2)$ & 0.682 & \\
\hline \multicolumn{5}{|l|}{ Symptoms } \\
\hline Fever, $n(\%)$ & $15(65.2)$ & $8(72.2)$ & 1.000 & \\
\hline Cough, n (\%) & $12(52.2)$ & $6(54.5)$ & 0.897 & \\
\hline Shortness of breath, $n$ (\%) & $12(52.2)$ & $9(81.8)$ & 0.140 & \\
\hline Myalgias, n (\%) & $4(17.4)$ & $0(0.0)$ & 0.280 & \\
\hline Gastrointestinal symptoms, n (\%) & $3(13.0)$ & $1(9.1)$ & 1.000 & \\
\hline \multicolumn{5}{|l|}{ Admission laboratory data } \\
\hline Leucocyte count, x10E3/uL, median (IQR) & $5.2(3.8-6.9)$ & $7.7(6.8-9.0)$ & 0.004 & $0.26[0.03-0.26]$ \\
\hline Lymphocyte count, /uL, median (IQR) & $660(460-1300)$ & $990(770-1370)$ & 0.329 & \\
\hline Neutrophil count, x10E3/uL, median (IQR) & $4.0(2.6-4.9)$ & $6.5(4.6-7.7)$ & 0.008 & $1.00[1.00-1.00]$ \\
\hline Platelet count, $\mathrm{x} 10 \mathrm{E} 3 / \mathrm{uL}$, mean $\pm \mathrm{SD}$ & $145.4 \pm 81.4$ & $193.9 \pm 37.1$ & 0.083 & $1.03[1.00-1.10]$ \\
\hline Aspartate transaminase, $\mathrm{U} / \mathrm{L}$, median (IQR) & $33.5(22.0-46.8)$ & $26.0(21.0-35.0)$ & 0.914 & \\
\hline Alanine transaminase, $\mathrm{U} / \mathrm{L}$, median (IQR) & $20(13.3-30.3)$ & $22(8-25)$ & 0.563 & \\
\hline LDH, U/L, median (IQR) & $253(220.5-325.8)$ & $263(228-325)$ & 1.000 & \\
\hline CRP, mg/dL, median (IQR) & $14.3(5.3-29.2)$ & $14.6(12.9-16.4)$ & 0.840 & \\
\hline Procalcitonin, ng/mL, median (IQR) & $1.3(1.1-1.3)$ & $2.0(1.8-2.1)$ & 0.947 & \\
\hline Ferritin, ng/mL, median (IQR) & $1699(1337-10886)$ & $1752(872-26-32)$ & 0.571 & \\
\hline D-dimer, ug/mL, median (IQR) & $2.7(1.63-4.0)$ & $6.5(6.20-6.50)$ & 0.295 & \\
\hline PT, seconds, median (IQR) & $15.7(11.8-57.8)$ & $16.4(15.7-17.0)$ & 0.284 & \\
\hline Troponin T, ng/mL, median (IQR) & $188.5(63.0-360.5)$ & $128(123.0-133.0)$ & 0.657 & \\
\hline CK, U/L, median (IQR) & $133(68.0-255.5)$ & $68(35.0-101.0)$ & 0.585 & \\
\hline \multicolumn{5}{|l|}{ Radiology findings } \\
\hline Unilateral pneumonia. $\mathrm{n}(\%)$ & $3(13.0)$ & $1(9.1)$ & 1.000 & \\
\hline Bilateral pneumonia. $n$ (\%) & $2(8.7)$ & $1(9.1)$ & 1.000 & \\
\hline \multicolumn{5}{|l|}{ Treatment } \\
\hline Hydroxychloroquine. n (\%) & $10(43.5)$ & $7(63.6)$ & 0.271 & \\
\hline Antiviral (Lopinavir/ritonavir or Remdesivir). $\mathrm{n}$ (\%) & $3(13.0)$ & $2(18.2)$ & 1.000 & \\
\hline Glucocorticoid, n (\%) & $2(8.7)$ & $2(18.2)$ & 0.580 & \\
\hline \multicolumn{5}{|l|}{ Clinical outcomes } \\
\hline Bacterial superinfection, $\mathrm{n}(\%)$ & $4(17.4)$ & $9(81.8)$ & 0.001 & $0.01[0.00-0.26]$ \\
\hline Hospitalization time (days), median (IQR) & $12(6-17)$ & $10(5-16)$ & 0.839 & \\
\hline Admission to ICU, n (\%) & $5(11.1)$ & $1(4.3)$ & 0.656 & \\
\hline
\end{tabular}

CK - creatinine kinase; CRP - C reactive protein; HD - hemodialysis; ICU - intensive care unit; IQR - interquartile range; LDH - lactate dehydrogenase; PT - prothrombin time; RAAS - renin angiotensin aldosterone SD - standard deviation. 


\section{Figure 1}

Kaplan-Meier survival curves for maintenance hemodialysis and control group.

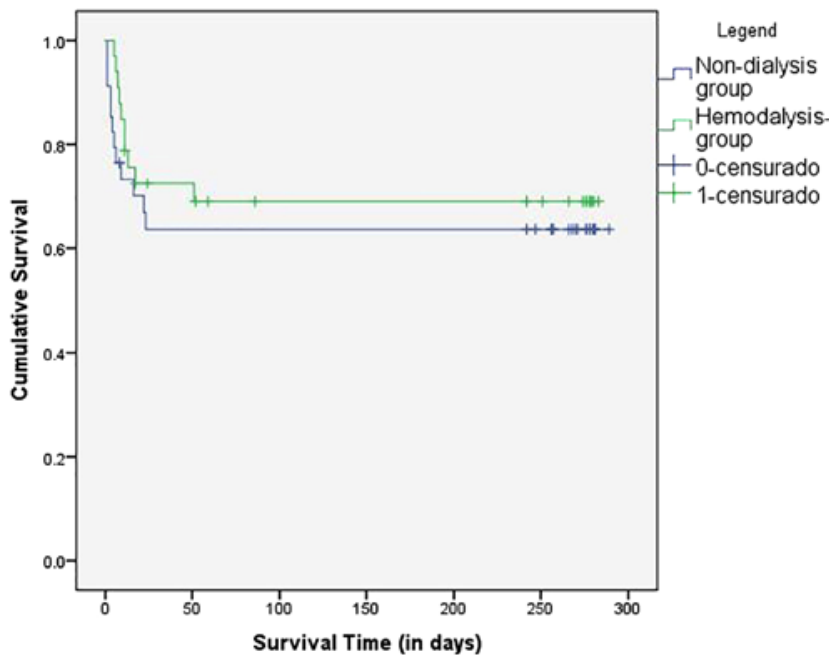

\section{DISCUSSION}

To our best knowledge, this is the first Portuguese study comparing outcomes of HD patients and propensity matched non-dialysis patients infected with COVID-19.

COVID-19 symptoms in the HD group were the same as in the control group. The earlier published reports found that HD patients presented with fewer symptoms and a milder course, whereas more recent studies have shown that clinical presentation is similar to the general population. . $^{-4,7-10}$ More patients in the HD group presented with respiratory insufficiency and needed supplementary oxygen. That could be explained by the contribution of hypervolemia, since acutely ill HD patients sometimes present weigh loss that could be not immediately noticed.

Comparing with the control group, patients in the HD group had higher procalcitonin, ferritin and troponin T levels. Procalcitonin is an early predictor of infection in HD patients and a predictor of bacterial infection. ${ }^{11,12}$ Although median procalcitonin levels were higher in the HD patients, there was similar rates of bacterial superinfection between groups. Some reports showed that chronic HD per se may affect procalcitonin levels. ${ }^{11-13}$ Ferritin could be a marker of systemic inflammation and higher levels are seen in chronic diseases. ${ }^{14}$ Therefore, basal levels of ferritin on HD patients could be higher and this could explain the difference between groups. Also, the finding of higher levels of $T$ troponin in the HD group was not unexpected. Baseline troponin T levels are higher in CKD patients, and when stable, do not signify acute myocardial infarction. Such elevations are thought to be related to the reduced clearance of such markers by the kidneys as well as due to chronic and persistent damage to cardiac myocytes via mechanisms associated with CKD. ${ }^{15}$ Transaminases were higher in the control group, also reported in another study. ${ }^{4}$ Serum aminotransferase levels are lower in HD patients, with or without viral hepatitis, compared to individuals with normal renal function. ${ }^{16}$
D-dimers and C reactive protein that are reported to be higher in HD patients were similar between groups. ${ }^{17,18}$ These findings question whether regular criteria for defining high risk COVID-19 patients should be applied to HD patients.

Unilateral pneumonia in the chest $\mathrm{X}$-ray was more frequent in the control group ( $11.8 \%$ vs $32.4 \%, p=0.041)$. These results may have some bias due to observer-dependent subjectivity on radiography observation.

There were no differences in both groups in terms of COVID-19 treatment and clinical outcomes. Glucocorticoid treatment was applied only to $11.8 \%$ of HD patients because the vast majority was admitted before the establishment of the dexamethasone protocol in our center. Although the use of antiviral therapy in HD patients is controversial, the control group had equally elderly patients with multiple comorbidities, which may also have constituted contraindications to those therapies. We also report low rates of ICU admission and this as well could be explained by the advanced age and high prevalence of comorbidities of both groups.

We report a mortality rate of $32.4 \%$ on HD patients which is in line with other studies that report in-hospital mortality on HD COVID-19 patients of $27 \%-32 \%$. ${ }^{2,8-10,19}$ We did not find differences in mortality between HD and control group. Recent studies also reported that the degree of disease severity and the mortality rate of dialysis patients was similar to an age and gender matched control population. ${ }^{3,8}$ Leukocytosis, neutrophilia, and bacterial superinfection were more common in HD patients who died during hospitalization. Shang $W$ et al. also reported that leucocytes and neutrophil count were predictive of a poor prognosis among maintenance HD patients infected with SARS-CoV-2. ${ }^{20}$ Although some studies have shown that lymphopenia is a predictor of disease severity in the general population, our results were not consistent with that. ${ }^{21}$ After adjusting for other confounders, only bacterial superinfection was a risk factor for mortality among dialysis patients. Other established risk factors for mortality in COVID-19 patients, such as age, diabetes, heart disease, or chronic obstructive lung disease were not associated with higher mortality in HD patients. $^{22,23}$

Several limitations of this study should be acknowledged. First, this is a single-center retrospective study with a small size sample, and some patients' laboratory and clinical data were incomplete. Second, we only included hospitalized patients and therefore patients with mild disease were excluded. A multi-center retrospective study should be conducted to include a larger sample size across multiple centers to better assess the effects of COVID-19 infections in HD patients.

\section{CONCLUSION}

Our study compared outcomes for COVID-19 patients on maintenance HD to non-dialysis patients with similar age, gender and comorbidities and showed no difference in hospital stay or death rate. Despite these results, we must emphasize that mortality in the dialysis group was exceptionally high, with up to $32 \%$ of in-hospital mortality, and that bacterial superinfection has been shown to be an independent 
predictor of mortality. Hence, we intend to highlight the importance of interventions, such as full vaccination coverage, to mitigate the burden of COVID-19 in HD patients.

Disclosure of potential conflicts of interest: none declared.

\section{References}

1. Ponto de Situação Atual em Portugal - COVID -19. Available at: https://covid19.min -saude.pt/ ponto -de -situacao -atual -em -portugal/ 8 [Accessed December 31, 2020].

2. Goicoechea M, Sánchez Cámara LA, Macías N, et al. COVID-19: clinical course and outcomes of 36 hemodialysis patients in Spain. Kidney Int. 2020; 98(1):27-34.

3. Chan L, Jaladanki SK, Somani S, et al. Outcomes of Patients on Maintenance Dialysis Hospitalized with COVID-19. Clin J Am Soc Nephrol. 2020;30:CJN.12360720.

4. Luo Y, Li J, Liu Z, Yu H, Peng X, Cao C. Characteristics and outcomes of hemodialysis patients with COVID-19: a retrospective single-center study. PeerJ. 2020; 26(8):e10459.

5. Rodriguez-Morales A, Cardona-Ospina J, Gutiérrez-Ocampo E, et al. Clinical, laboratory and imaging features of COVID-19: A systematic review and meta-analysis. Travel Medicine and Infectious Disease. 2020; 34.101623

6. Wang R, Liao C, He H et al. COVID-19 in Hemodialysis Patients: A report of 5 cases. American Journal of Kidney Diseases. 2020; 76(1):141-143.

7. Hsu C, Weiner D. COVID-19 in dialysis patients: outlasting and outsmarting a pandemic. Kidney international. 2020; 98:1402-1404.

8. $\mathrm{Ng} \mathrm{JH}$, Hirsch JS, Wanchoo R, et al. Northwell COVID-19 Research Consortium and the Northwell Nephrology COVID-19 Research Consortium: Outcomes of patients with end-stage kidney disease hospitalized with COVID-19 Kidney Int. 2020; 98(6):1530-1539.

9. Valeri AM, Robbins-Juarez SY, Stevens JS, et al. Presentation and outcomes of patients with ESKD and COVID-19. J Am Soc Nephrol. 2020; 31(7):1409-1415.

10. Tortonese S, Scriabine I, Anjou L, et al. COVID-19 in patients on maintenance dialysis in the Paris region, Kidney Int Rep. 2020; 5(9):1535-1544.

11. Demir NA, Sumer S, Celik G, et al. How should procalcitonin and C-reactive protein levels be interpreted in haemodialysis patients? Intern Med J. 2018; 48(10):1222-1228.

12. Quiroga B, Villaverdeb M, Vegaa A, Abada S, Requea J, López-Gómez JM. La procalcitonina como indicador temprano de infección aguda en pacientes en hemodiálisis. Nefrología. 2014; 34(3):341346.

13. Ichihara K, Tanaka T, Takahashi S. et al. Serum procalcitonin level in chronic hemodialytic patients with no evidence of bacterial infection. Ren Replace Ther. 2016; 2:9.

14. Kalantar-Zadeh K, Kalantar-Zadeh K, Lee GH. The fascinating but deceptive ferritin: to measure it or not to measure it in chronic kidney disease? Clin J Am Soc Nephrol. 2006; 1(1):9-18.
15. Levi M, Bonenfant F, Brouwers FM, Farand P, Corbin F, Nguyen M. Impact of hemodialysis on the level of high-sensitivity cardiac troponins $\mathrm{T}$ in patients with end-stage renal disease. Minerva Cardioangiol. 2015; 63(3):179-186.

16. Sette $L H$, Almeida Lopes EP. Liver enzymes serum levels in patients with chronic kidney disease on hemodialysis: a comprehensive review. Clinics (Sao Paulo). 2014; 69(4):271-278.

17. Gubensek J, Lolic M, Ponikvar R, Buturovic-Ponikvar J. D-dimer levels in maintenance hemodialysis patients: high prevalence of positive values also in the group without predisposing diseases. Hemodial Int. 2016 Apr; 20(2):198-203.

18. Demir NA, Sumer S, Celik G, Afsar RE, Demir LS, Ural O. How should procalcitonin and C-reactive protein levels be interpreted in haemodialysis patients? Intern Med J. 2018 Oct; 48(10):1222-1228.

19. Hilbrands LB, Duivenvoorden R, Vart P, et al. COVID-19-relted mortality in kidney transplant and dialysis patients: results of the ERACODA collaboration. Nephrol Dial Transplant. 2020; 35(11):1973-1983.

20. Shang $\mathrm{W}, \mathrm{Li} Y, \mathrm{Li} \mathrm{H}$, et al. Correlation between laboratory parameters on admission and outcome of COVID-19 in maintenance hemodialysis patients. Int Urol Nephrol. 2021;53(1):165-169.

21. Tan L, Wang $Q$, Zhang $D$, et al. Lymphopenia predicts disease severity of COVID-19: a descriptive and predictive study. Sig Transduct Target Ther. 2020; 5(1):33.

22. Du RH, Liang LR, Yang CQ, et al. Predictors of mortality for patients with COVID-19 pneumonia caused by SARS-CoV-2: a prospective cohort study. Eur Respir J. 2020; 55(5):2000524

23. Tian W, Jiang W, Yao J, et al. Predictors of mortality in hospitalized COVID-19 patients: a systematic review and meta-analysis. J Med Virol. 2020;92(10):1875-1883.

\section{ORCID}

Marina Reis iD 0000-0003-3061-3767

Catarina Almeida (iD) 0000-0001-6806-5597

Ana Ventura iD 0000-0002-7197-7797

Catarina Ribeiro iD 0000-0001-6813-0336

Ana Marta Gomes iD 0000-0002-8992-5792

Daniela Lopes (D) 0000-0002-1066-1676

Clara Santos iD 0000-0002-8616-5396

Sónia Sousa iD 0000-0003-0201-8282

João Carlos Fernandes (iD) 0000-0003-0581-3097

\section{Correspondence to:}

Marina Reis

R. Conceição Fernandes; 4434-502 Vila Nova de Gaia, Portugal

E-mail: marina.reis9@gmail.com 